\title{
Cognitive reserve moderates the association between functional network anti-correlations and memory in $\mathrm{MCI}$
}

\author{
Nicolai Franzmeier ${ }^{a}$, Katharina Buerger ${ }^{\mathrm{a}}$, Stefan Teipel ${ }^{\mathrm{b}, \mathrm{c}}$, Yaakov Stern ${ }^{\mathrm{d}}$, \\ Martin Dichgans ${ }^{\mathrm{a}, \mathrm{e}, \mathrm{f}}$, Michael Ewers ${ }^{\mathrm{a}, *}$, for the Alzheimer's Disease Neuroimaging \\ Initiative (ADNI) \\ a Institute for Stroke and Dementia Research, Klinikum der Universität München, Ludwig-Maximilians-University LMU, Munich, Germany \\ ${ }^{\mathrm{b}}$ Department of Psychosomatic Medicine, University of Rostock, Rostock, Germany \\ ${ }^{\mathrm{c}}$ German Center for Neurodegenerative Diseases (DZNE, Rostock), Rostock, Germany \\ ${ }^{\mathrm{d}}$ Cognitive Neuroscience Division, Department of Neurology, Columbia University College of Physicians and Surgeons, New York, NY, USA \\ ${ }^{\mathrm{e}}$ Munich Cluster for Systems Neurology (SyNergy), Munich, Germany \\ ${ }^{\mathrm{f}}$ German Center for Neurodegenerative Diseases (DZNE, Munich), Munich, Germany
}

\section{A R T I C L E I N F O}

\section{Article history:}

Received 30 June 2016

Received in revised form 14 November 2016

Accepted 19 November 2016

Available online 1 December 2016

\section{Keywords:}

Sliding time window functional connectivity Resting-state fMRI

Cognitive reserve

Mild cognitive impairment

Memory

\begin{abstract}
A B S T R A C T
Cognitive reserve (CR) shows protective effects on cognitive function in older adults. Here, we focused on the effects of $\mathrm{CR}$ at the functional network level. We assessed in patients with amnestic mild cognitive impairment ( $\mathrm{aMCl}$ ) whether higher $\mathrm{CR}$ moderates the association between low internetwork cross-talk on memory performance. In 2 independent aMCI samples ( $\mathrm{n}=76$ and 93 ) and healthy controls $(\mathrm{HC}, \mathrm{n}=36), \mathrm{CR}$ was assessed via years of education and intelligence (IQ). We focused on the anti-correlation between the dorsal attention network (DAN) and an anterior and posterior default mode network (DMN), assessed via sliding time window analysis of resting-state functional magnetic resonance imaging (fMRI). The DMN-DAN anti-correlation was numerically but not significantly lower in aMCI compared to HC. However, in aMCI, lower anterior DMN-DAN anti-correlation was associated with lower memory performance. This association was moderated by CR proxies, where the association between the internetwork anti-correlation and memory performance was alleviated at higher levels of education or IQ. In conclusion, lower DAN-DMN cross-talk is associated with lower memory in aMCI, where such effects are buffered by higher CR.
\end{abstract}

(c) 2016 Elsevier Inc. All rights reserved.

\section{Introduction}

Cognitive reserve (CR) designates the ability to cognitively perform relatively well in the presence of neuropathology and is thought to be conferred by higher mental abilities like IQ or prolonged life-time experiences such as education. The CR hypothesis is supported by findings from epidemiologic studies showing that proxy measures of CR including IQ and years of formal education are associated with lower risk of dementia (Esiri and Chance, 2012; Meng and D'Arcy, 2012; Valenzuela and Sachdev, 2006; Wilson et al., 2002). Further support for the CR hypothesis comes from neuroimaging studies on mild cognitive impairment (MCI) and Alzheimer's disease (AD), which showed that patients with higher levels of $C R$ proxy measures exhibited stronger neuropathology (such as amyloid pathology, gray matter atrophy, glucose hypometabolism) at relatively

\footnotetext{
* Corresponding author at: Institute for Stroke and Dementia Research, Klinikum der Universität München, Feodor-Lynen-Strasse 17, 81377 Munich, Germany. Tel.: +49 894400 46221; fax: +49 (0)894400 46113 .

E-mail address: michael.ewers@med.uni-muenchen.de (M. Ewers).
}

stable levels of cognitive performance (Ewers et al., 2013; Morbelli et al., 2013; Stern, 2006; Vemuri et al., 2011). CR-related effects at the level of functional brain networks, however, are not well understood (Barulli and Stern, 2013). Since previous fMRI studies showed circumscribed changes in functional networks that are linked to cognitive impairment in patients with $\mathrm{MCI}$ (for review see (Teipel et al., 2016)), the question arises whether higher levels of CR buffer the effects of lower neural network function on memory in $\mathrm{MCl}$. Motivated by a previous finding in nondemented older adults, which showed that at higher levels of IQ the association between amyloid pathology and episodic memory impairment was weaker (Rentz et al., 2010), we aimed to test whether at higher levels of CR (as measured by IQ or education), lower levels of functional connectivity between functional brain networks is associated with relatively milder memory impairment in $\mathrm{MCI}$.

To assess functional network changes that underlie memory impairment, we measured resting-state functional connectivity between 2 major functional networks including the dorsal attention network (DAN) and the default mode network (DMN). Previous resting-state fMRI studies have revealed an anti-correlation between 
the resting-state signal of the DMN and the DAN (Carbonell et al., 2014; Chai et al., 2014; Fox et al., 2005), where greater DMN-DAN anti-correlation was associated with higher cognitive control and better working memory performance (Anticevic et al., 2012; Chai et al., 2014; Hampson et al., 2010; Kelly et al., 2008). The restingstate DMN-DAN anti-correlation is reminiscent of observations from task-related fMRI studies showing a task activation of the DAN concomitant with a deactivation of the DMN to be associated with higher episodic memory performance in cognitively normal individuals (Kim et al., 2010; Kragel and Polyn, 2015; Landsiedel and Gilbert, 2015). Here, we hypothesized that in amnestic MCI-a clinical syndrome characterized by memory impairment-lower levels of the DMN-DAN anti-correlation are associated with low memory performance and that such an association can be buffered by CR. Although the anti-correlation of the DAN and DMN itself was not pathologically altered before the stage of $A D$ dementia in previous studies (Wang et al., 2007; Zhu et al., 2016), we hypothesized that the level of the anti-correlation of the DMN-DAN is predictive of memory impairment in the diseased brain $(\mathrm{MCI})$ at low levels of CR. The rationale is that the reciprocal activation of the DAN and DMN has a central regulating role within the ensemble of functional networks underlying cognition (Cole et al., 2014), which becomes especially crucial to maintain successful cognitive processes including memory at the stage of $\mathrm{MCI}$ and low $\mathrm{CR}$.

To assess the DAN-DMN anti-correlation, we applied functional connectivity analysis of the DMN-DAN anti-correlation in 2 independent samples of amnestic MCI patients for cross-validation of the results. Via sliding time window functional connectivity analysis, we identified episodes of peak anti-correlations between the networks during resting-state fMRI (Allen et al., 2014; Chang and Glover, 2010; Hutchison et al., 2013a; Sadaghiani et al., 2015; Zalesky et al., 2014). Subsequently, we tested the hypothesis that higher levels of CR proxies (IQ or years of education) moderate the relationship lower levels of peak DMN-DAN anti-correlation and memory impairment in $\mathrm{MCl}$. We included patients with the clinical syndrome of amnestic MCI patients, both single- and multipledomain amnestic types, regardless of underlying etiology. To assess whether presence of AD pathology (increased levels of amyloid PET) or genetic risk of $\mathrm{AD}$ (ApoE $\varepsilon 4$ genotype) influence the effect of CR proxies on the relationship between DMN-DAN anticorrelation and memory impairment, we controlled for both amyloid PET levels and APOE genotype.

\section{Methods}

\subsection{Study sample}

A total of 76 patients with amnestic $\mathrm{MCI}(\mathrm{MCI})$ and 36 healthy older adults (HC) were included from the Alzheimer's Disease Neuroimaging Initiative (ADNI) (Petersen et al., 2010). For crossvalidation purposes, we included a sample of 93 patients with $\mathrm{MCI}$, who were recruited at the memory clinic of the Institute for Stroke and Dementia Research (ISD), Klinikum der Universitaet Muenchen, Munich (henceforth referred to as the ISD sample).

In the ADNI study, MCI was diagnosed according to the Petersen (Petersen, 2004) criteria. MCI patients showed Mini Mental State Examination (MMSE) scores between 24 and 30 (inclusive), subjective and objective memory loss as defined by scores 1.5 SD below age and education adjusted scores on the Wechsler Memory Scale Logical Memory II, a CDR of 0.5, and absence of significant levels of impairment on other cognitive domains. An individual was defined as $\mathrm{HC}$ when showing an MMSE score between 24 and 30 (inclusive), a CDR of 0 , no signs of depression, and no objective memory loss (for further details on diagnostic guidelines and neuropsychological examinations please see the ADNI study website (https://adni.loni.usc.edu/wp-content/ uploads/2010/09/ADNI_GeneralProceduresManual.pdf)).

Beyond the inclusion criteria defined by ADNI, additional requirements for the inclusion in the present study had to be met, consisting of the availability of resting-state fMRI, T1-weighted MRI images, [18-F] AV-45 amyloid PET and comprehensive neuropsychological test scores. Amyloid beta $(A \beta)$ levels in the brain were determined based on a global measure of [18-F] AV-45 PET binding, which is provided by the ADNI PET core as a summary measure of several cortical ROIs that have been described previously (Landau et al., 2013). Specifically, an abnormally high $A \beta$ level was defined according to an a priori established cutoff value (Landau et al., 2013), i.e., a global AV-45 PET binding (SUVR) $\geq 1.11(A \beta+, n=44$ for MCI; $n=13$ for $\mathrm{HC}$ ). Normal low $A \beta$ levels (AV-45 PET binding [SUVR] $<1.11, A \beta-$ ) were present in $32 \mathrm{MCI}$ and $23 \mathrm{HC}$.

In the ISD study, MCI was also diagnosed according to the Petersen (Petersen, 2004) criteria. MCI was defined as a score 1.5 SD below the norm (adjusted for age, education) on at least one of the memory subtests (word list or figure learning/recall) of the CERAD-Plus battery (Luck et al., 2009). All MCI patients underwent resting-state fMRI, T1-weighted MRI, and comprehensive neuropsychological testing. Additional inclusion criteria consisted of age $>55$ years, absence of symptoms of depression, other neurological disorders, uncontrolled arterial hypertension or diabetes mellitus or a history of alcohol or drug abuse. The ISD-MCI patients had been recruited for a cognitive intervention trial at the ISD [registered at www.clinicaltrials.gov as NCT01525368 as "Outcome Predictors of a Cognitive Intervention in aMCl”; see (Franzmeier et al., 2016) for details]. However, for the present study, we used only baseline data that were assessed before the cognitive intervention.

\subsection{Standard protocol approvals, patient consents, and registration}

For the ADNI sample, ethical approval was obtained by the ADNI investigators (for details see: http://www.adni-info.org/pdfs/adni_ protocol_9_19_08.pdf). Recruitment of the ISD sample was approved by the ethics committee of the Ludwig Maximilian University Munich. All procedures were conducted in accordance with the Helsinki Declaration of 1975 and the applicable revisions at the time of the investigation. Written informed consent was obtained from all patients for being included in the study.

\subsection{Neuropsychological assessment}

To estimate memory performance, we used the ADNI-MEM score, a composite measure of episodic memory based on a broad battery of neuropsychological memory tests, as described previously for the ADNI study (Crane et al., 2012). Briefly, the ADNI-MEM score takes into account scores of the Rey Auditory Verbal Learning Test, AD Assessment Scale-Cognitive Subscale, word recall (3 words) of the MMSE and the Wechsler Logical Memory Scale II.

For the ISD-Munich sample, we generated a memory composite score via a similar approach that was used for the ADNI-MEM score (Crane et al., 2012). Here, we selected cognitive tests that are similar regarding test construction and measure the same neuropsychological construct (i.e., memory). To this end, we used the verbal memory subtests of the CERAD-Plus battery (Luck et al., 2009), the learning and recall subscales of the German version of the California Verbal Learning Test (Woods et al., 2006), and the word list learning and recall subtest of the AD Assessment Scale (Rozzini et al., 2008). Next, we applied maximum-likelihood confirmatory factor analysis to these memory tests, using a single factor model. The analysis yielded factor weights for each memory test loading on a principal factor with an eigenvalue of 9.14, indicating that the composite score captures considerably more variance than a single measure. For each 
subject, the factor score was computed and z-score transformed to be used as a summary memory index in the subsequent analyses.

\subsection{CR assessment}

For the ADNI study, we employed years of formal education and premorbid verbal IQ [as assessed via the American national adult reading test; ANART (Bright et al., 2002)] as commonly used CR proxies (Stern, 2012). For the ISD study, we applied the premorbid verbal IQ assessed via the German multiple vocabulary test (Hessler et al., 2013; Valenzuela and Sachdev, 2006). We did not use years of education in the ISD sample because many older patients in Germany experienced their educational years during or shortly after World War II, which did compromise the educational system and may limit the reliability of the number of years of education as a proxy of CR. Thus, we focused on IQ as a CR proxy, whose scoring procedure is independent of the number of years of education. Since both education and IQ are frequently used proxies of CR and have been shown to be correlated (Nucci et al., 2012; Tucker and Stern, 2011), they both relate to $\mathrm{CR}$. Hence, the interpretation of results should be similar.

\subsection{Data acquisition}

In the ADNI study, scanning was performed on Philips 3T MRI scanners, using an 8-channel head matrix coil. T1-weighted anatomical images were acquired using a 3-dimensional (3D) MPRAGE sequence, with whole brain coverage and $1 \times 1 \times 1.2 \mathrm{~mm}$ voxel resolution. Resting-state fMRI images were recorded using a single shot $\mathrm{T}^{*}$-weighted echo planer imaging (EPI) pulse sequence in transverse slice orientation, with a repetition time of $3000 \mathrm{~ms}$, a flip angle of $80^{\circ}$, and $3.3-\mathrm{mm}$ isotropic voxel resolution. The restingstate fMRI sequence comprised a total number of 140 volumes.

In the ISD study, scanning was performed on a Siemens 3T Magnetom Verio Scanner with a 12-channel head matrix coil. T1weighted anatomical images were acquired using a 3D MPRAGE sequence with whole brain coverage and an isotropic voxel resolution of $1 \mathrm{~mm}$. Resting-state fMRI images were acquired using a T2*-weighted EPI pulse sequence in transverse slice orientation with a repetition time of $3000 \mathrm{~ms}$, a flip angle of $80^{\circ}, 3-\mathrm{mm}$ isotropic voxel resolution, and a total number of 120 volumes.

\subsection{Preprocessing and spatial normalization of resting state fMRI data}

All preprocessing steps were conducted using SPM 12 (Wellcome Trust Centre for Neuroimaging, University College London). The same preprocessing protocol, if not otherwise noted, was applied to the resting-state fMRI scans from ADNI and ISD. For each subject, the first 10 volumes of the fMRI time series were discarded because of known instabilities of the MR signal at the beginning of the fMRI acquisition. The remaining volumes were realigned to the first volume, motion corrected, and coregistered to native space 3D T1-weighted images and smoothed using a Gaussian kernel with a full width at half maximum of $8 \mathrm{~mm}$. Preprocessed resting-state fMRI images were then spatially normalized using DARTEL (Ashburner, 2007), where the spatial normalization parameters and the creation of customized gray matter templates were done separately for the ADNI and ISD groups to avoid biases due to scanner differences between both studies. However, the same, spatial normalization protocol was used for both ADNI and ISD. In a first step, high-resolution 3D T1-weighted images were segmented in gray matter (GM), white matter (WM), and cerebrospinal fluid (CSF) maps, using SPM's new-segment approach (Ashburner and Friston, 2005). Based on the segmentations, we applied a diffeomorphic high-dimensional registration algorithm to warp individual subject brains to a common space that is defined in an iterative procedure, as implemented in the SPM 12 toolbox DARTEL (Ashburner, 2007). The resulting gray matter group template was then affine registered to the Montreal Neurological Institute (MNI) template of T1 images implemented in DARTEL.

The DARTEL flow fields and affine registration transformation matrix were subsequently combined and applied to each subjects' coregistered fMRI volumes for spatial normalization to the MNI template.

To remove noise from the EPI images, the spatially normalized resting-state fMRI images were detrended and band-pass filtered with a frequency band of $0.01-0.08 \mathrm{~Hz}$. In addition, we regressed out the 6 motion parameters and the BOLD signal averaged across the WM and CSF. Since we were specifically interested in anticorrelations between DMN and DAN, we did not perform global signal regression, which may artificially introduce anti-correlations between resting-state fMRI signal changes of the DMN and DAN (Carbonell et al., 2014; Chai et al., 2012; Murphy et al., 2009; Spreng et al., 2016). To create GM masks, we averaged spatially normalized but unmodulated GM maps, followed by binarization (voxel value $>0.3$ ), which was conducted separately within the ADNI and the ISD groups.

For the extraction of GM volume, we additionally created spatially normalized GM maps for each subject, that were smoothed with an 8-mm full width at half maximum Gaussian kernel, following a previously described approach (Mak et al., 2011). During the normalization step, modulation was applied so that local concentrations of gray matter are preserved after warping the image to template space.

\subsection{Resting-state fMRI data analysis}

\subsubsection{Independent component analysis}

Spatial group ICA (Calhoun and Adali, 2012) on preprocessed resting-state fMRI data was performed using the Infomax Algorithm implemented in the GIFT-Toolbox (http://mialab.mrn.org/ software/gift). The ICAs of resting-state fMRI scans were run separately for the ADNI and ISD samples. The number of independent components (ICs) to extract was a priori set to 20, which has previously been shown to robustly identify the DMN and DAN (Di and Biswal, 2014). To estimate the reliability of the resulting IC decomposition, the Infomax Algorithm was repeated 10 times in ICASSO (http://research.ics.aalto.fi/ica/icasso). All components showed stability indices greater than 0.95 in both groups suggesting that the components extracted by the group ICA algorithm show a high reliability. In line with earlier studies (Damoiseaux et al., 2012; Di and Biswal, 2014; Jones et al., 2011, 2012; Smith et al., 2009), we found the DMN to be subdivided into an anterior (aDMN) and a posterior (pDMN) component in both ADNI and ISD samples (see Fig. 1). The identification of the networks was confirmed by spatial regression matching the group ICA maps to the IC templates of major resting state networks (Laird et al., 2011). After group ICA, subject-specific IC maps and time courses (TCs) for the aDMN, pDMN, and DAN were back-reconstructed using GICA3 algorithm (Erhardt et al., 2011).

\subsubsection{Sliding time window analysis of network time courses}

We applied sliding time window analysis on the ICA derived component time courses to estimate peaks of anti-correlation between the aDMN versus DAN pair and the pDMN versus DAN pair (See Fig. 2 for illustration of the analysis). To this end, we used the GIFT-Toolbox (Allen et al., 2014), applying a sliding time window with a width of 30 repetition time (TR)'s (equivalent to 90 seconds of MRI acquisition) shifted in steps of 1 TR on the time courses of the 3 ICs. In line with previous studies, the rectangular time 


\section{Group Components}

anterior DMN
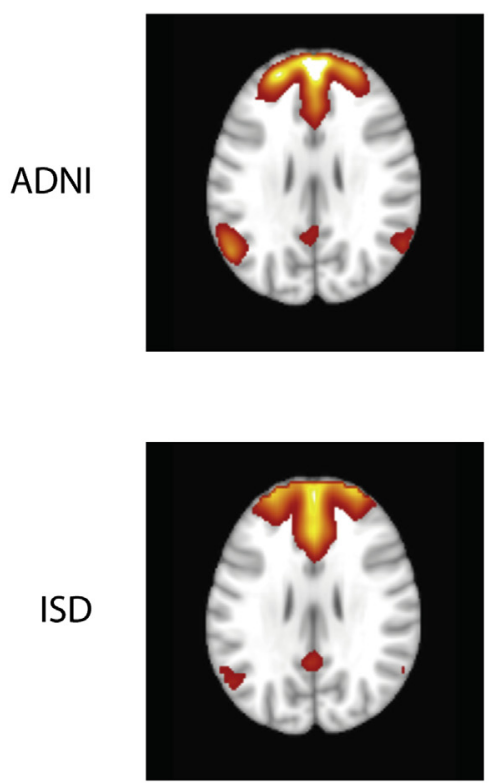

posterior DMN
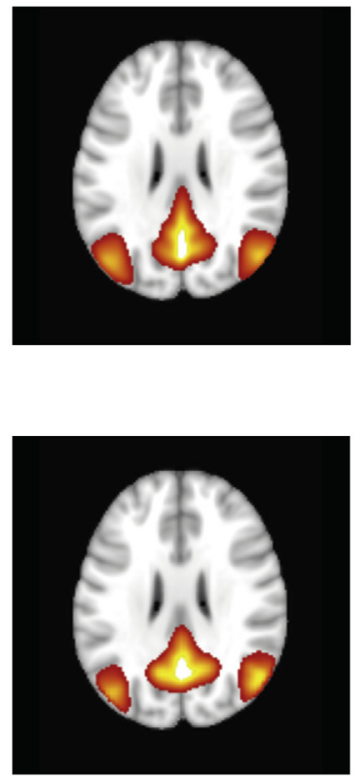

DAN
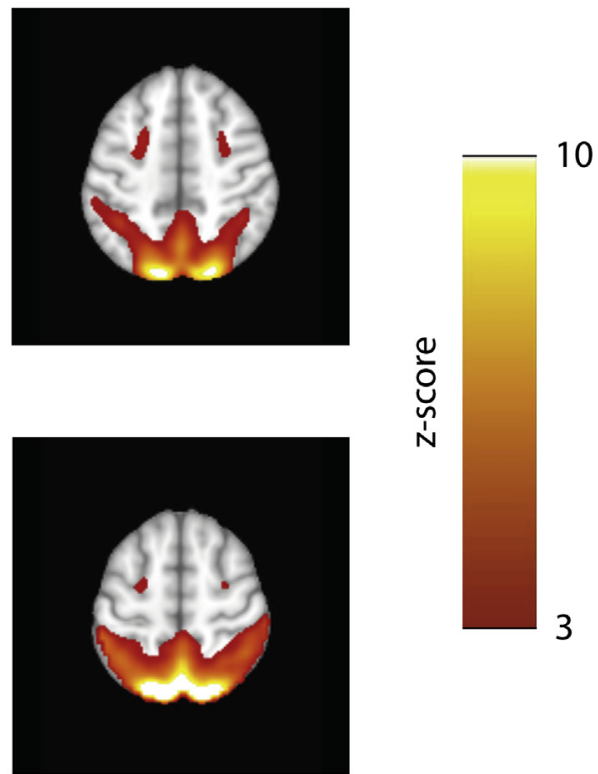

3

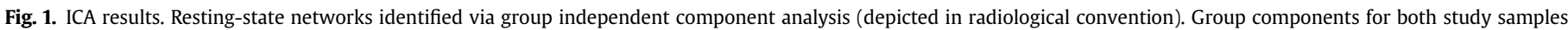

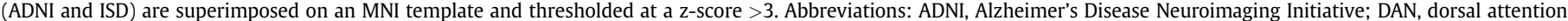
network; DMN, default mode network; ICA, independent component analysis; ISD, Institute for Stroke and Dementia Research.

window was convolved with a Gaussian sigma of 3 TRs to achieve tapering near the edges (Allen et al., 2014). Within each time window, we computed the Pearson moment correlation between the ICA time courses of each DMN component and the DAN. Sliding the window successively yielded a continuous graph reflecting internetwork correlations over the entire duration of the restingstate scan for each subject (Fig. 2). As a measure of peak anticorrelation between the networks, we computed the mean correlation coefficient across those consecutive 10 time windows (equivalent to 40 TR's or 120 seconds of MRI acquisition) that surrounded the time window showing the highest anti-correlation between the networks' time courses. To test the impact of time window selection on our findings, we conducted all analyses thrice, using the mean of the 5 or 15 time windows (equivalent to 105 and 135 seconds of resting-state fMRI) showing the highest negative correlations. Window selection did not change the result pattern of our statistical analyses testing our hypothesis as reported in the following. We further aimed to assess whether changes in peak functional connectivity were confounded by spontaneous head motion (Power et al., 2012). To this end, we computed the framewise displacement (FD) for each subjects' resting-state scan, following a previously described approach (Power et al., 2012). In brief, the FD indexes the relative head movement between subsequent EPI volumes and is calculated as the sum of the absolute values of the differentiated realignment estimates at every timepoint. Subsequently, we computed the overall mean FD, the mean FD within those time windows encompassing the peak anticorrelations as well as the mean FD of all remaining time windows.

\subsubsection{Assessment of static within- and between-network functional connectivity (FC)}

As a validation measure to the peak anti-correlation assessed via the sliding time window approach, we computed the more common between-network connectivity averaged across the whole resting-state scan similar to previous studies (Fox et al., 2005; Uddin et al., 2009). To this end, we assessed the Pearson moment correlation between the ICA-derived component whole-scan time courses of each DMN component and the DAN component. To later address confounding effects of head motion, we also computed the mean FD averaged across the whole resting-state scan. To extract the specific mean stationary FC within the network components for each individual, we binarized the group component maps of the aDMN, pDMN, and DAN at a threshold of $\mathrm{z}$-score $>3$. The binarized IC masks were then superimposed onto each individuals' respective IC map to compute a mean z-score transformed independent component value for each network and individual, reflecting intranetwork FC.

\subsection{GM volumetric assessment}

To control for potentially confounding effects of GM atrophy in the analysis of the resting-state anti-correlation between a(p)DMN and DAN, we computed the gray matter volume within the spatial intersection between the binary GM mask and binarized (threshold $z>3$ ) aDMN-DAN and pDMN-DAN maps. Specifically, we extracted the aDMN-DAN and pDMN-DAN GM volume for each subject from the modulated, smoothed, and normalized GM maps that were created during the preprocessing of structural MRI images. All volumetric measures were subsequently adjusted to intracranial volume (i.e., the sum of CSF, WM, and GM maps).

\subsection{Statistical analysis}

Demographics between all study groups were compared using $t$ tests for continuous variables and chi-squared test for categorical variables. Next, we assessed in the ADNI sample, whether the peak 


\section{A Identification of Resting State Networks}

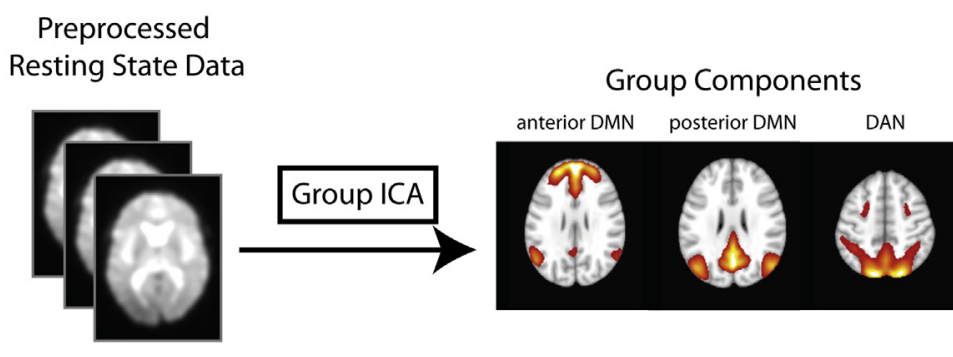

B Sliding Time-Window Functional Connectivity Analysis
Back reconstruction of subject specifc timecourses

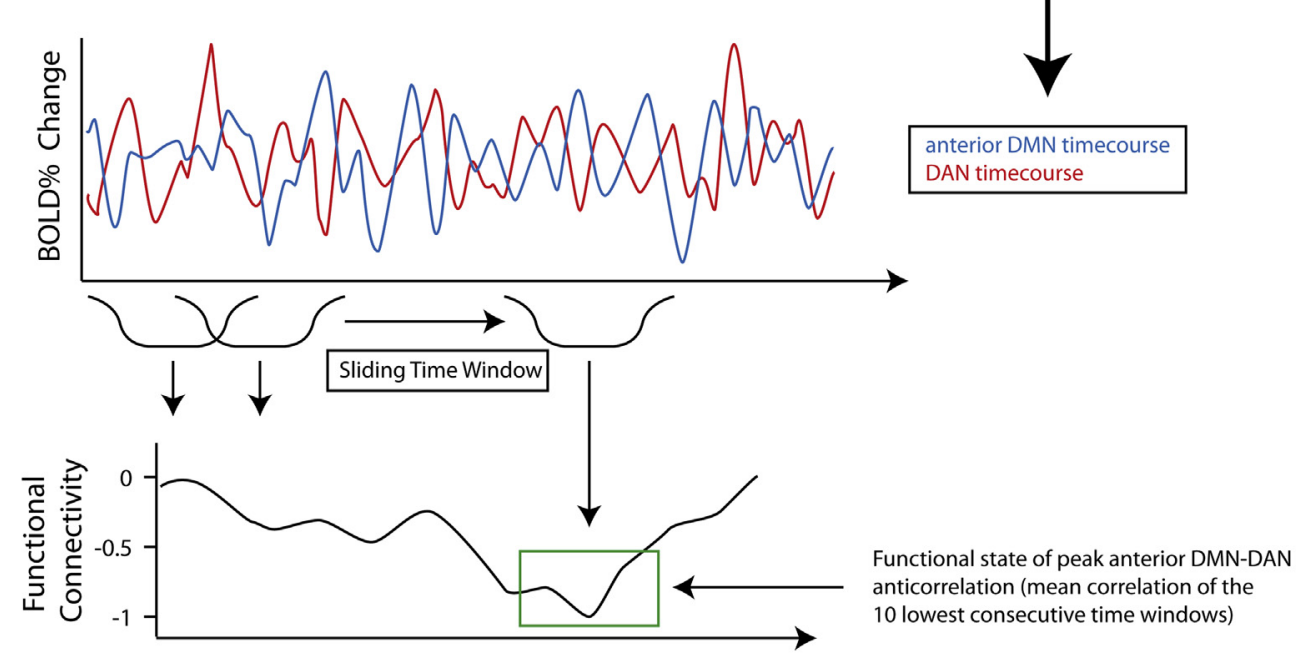

Time

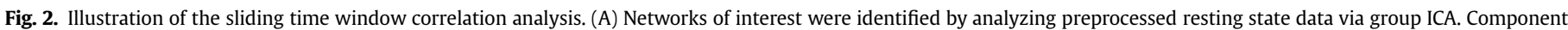

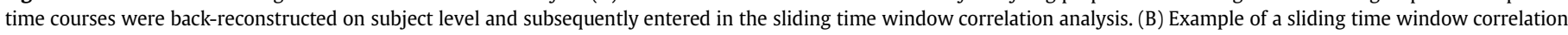

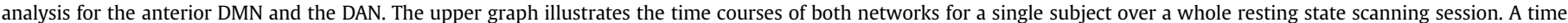

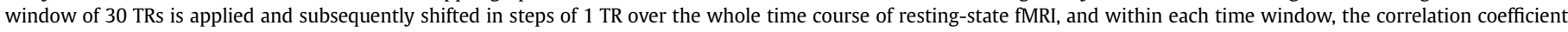

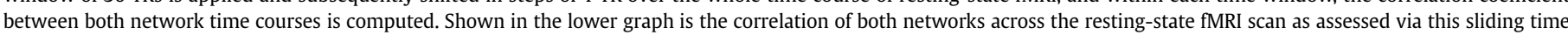

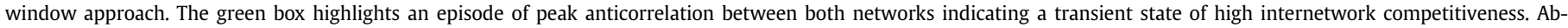

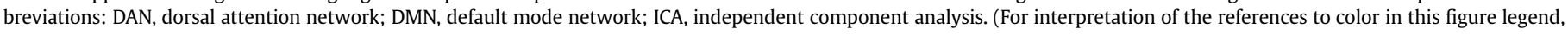
the reader is referred to the Web version of this article.)

aDMN-DAN or pDMN-DAN anti-correlations (henceforth referred to as a(p)DMN-DAN anti-correlation) were pathologically reduced in $\mathrm{MCI}$, using analysis of covariance with group (MCI vs. $\mathrm{HC}$ ) as independent variable, controlling for age, gender, and education.

To address our main hypothesis that $C R$ proxies moderate the relationship between the a(p)DMN-DAN anti-correlation and memory, we applied all regression analyses described below separately for the peak aDMN-DAN and peak pDMN-DAN anticorrelations. In all interaction models, CR proxies and the peak aDMN-DAN anti-correlation were entered as continuous measures. For illustrational purposes, interactions are, however, visualized by binarizing CR groups via median split.

For the ADNI sample, we tested the main and interaction effect of $\mathrm{CR} \times$ peak a(p)DMN-DAN anti-correlation on ADNI-MEM (memory composite), with additional covariates including age, gender, AV-45 PET status ( $A \beta+$ vs $A \beta-$ ), a(p)DMN-DAN GM volume and mean FD within the time windows of peak a(p)DMN-DAN anti-correlation. To investigate whether moderating effects of $C R$ were different for $A \beta$ positive or negative MCI patients, we also tested the 3-way interaction effect of AV-45 PET status $\times$ peak a(p) DMN-DAN anti-correlation $\times$ CR on the ADNI-MEM score, controlling for age and gender and a(p)DMN-DAN GM volume and mean FD within the time windows of peak a(p)DMN-DAN anticorrelation. To assess whether results were specific for peak functional connectivity, we conducted confirmatory analyses using the static whole time course instead of the peak a(p)DMN-DAN anti-correlation. For cross-validation purposes, we applied the same regression analyses to the $\mathrm{MCI}$ patients of the ISD study. Although $A \beta$ levels were not assessed in the ISD sample, we included the Apolipoprotein ( $A P O E$ ) genotype (i.e., $\varepsilon 4$ allele carrier vs. non-carrier) as a covariate indexing an individuals' risk of presence of AD pathology.

To assess whether stronger reductions in the peak a(p)DMN-DAN anti-correlation were driven by $\mathrm{AD}$-specific brain changes 
Table 1

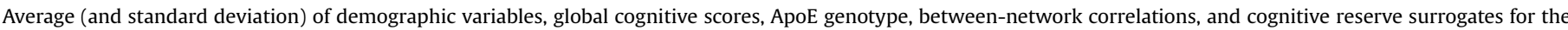
ADNI and ISD samples

\begin{tabular}{|c|c|c|c|}
\hline & ADNI-HC & ADNI-MCI & ISD-MCI \\
\hline Sample size & 36 & 76 & 93 \\
\hline Age & $75(6.3)$ & $71(7.5)^{\mathrm{a}}$ & $73.8(5.7)$ \\
\hline Gender $\mathrm{f} / \mathrm{m}$ & $21 / 15$ & $36 / 40$ & $43 / 50$ \\
\hline MMSE & $28.8(1.2)$ & $27.9(1.8)$ & $27.3(1.8)$ \\
\hline \multicolumn{4}{|l|}{ MCI subtype } \\
\hline Amnestic single-/multiple-domain & n.a. & $67 / 9^{\mathrm{b}}$ & $35 / 58$ \\
\hline \multicolumn{4}{|l|}{$A P O E$ status } \\
\hline$\varepsilon 4$ carriers/noncarriers & $12 / 24$ & $33 / 43$ & $50 / 43$ \\
\hline \multicolumn{4}{|l|}{ AV-45 status } \\
\hline $\mathrm{A} \beta-\mid \mathrm{A} \beta+$ & $23 / 13$ & $32 / 44^{a}$ & n.a. \\
\hline \multicolumn{4}{|l|}{ DMN-DAN correlation } \\
\hline aDMN-DAN & $-0.48(0.5)$ & $-0.48(0.4)$ & $-0.41(0.4)$ \\
\hline aDMN-DAN (age adjusted) & $-0.51(0.5)$ & $-0.47(0.4)$ & n.a. \\
\hline pDMN-DAN & $-0.60(0.4)$ & $-0.58(0.4)$ & $-0.57(0.4)$ \\
\hline pDMN-DAN (age adjusted) & $-0.61(0.4)$ & $-0.59(0.4)$ & n.a. \\
\hline \multicolumn{4}{|l|}{ Cognitive reserve surrogate } \\
\hline Years of education & $16.19(2.1)$ & $16.39(2.6)$ & n.a. \\
\hline ANART (number of errors) & $7.56(5.48)$ & $12.83(9.7)^{\mathrm{a}}$ & n.a. \\
\hline MWT-B (IQ) & n.a. & n.a. & $116.68(13.7)$ \\
\hline
\end{tabular}

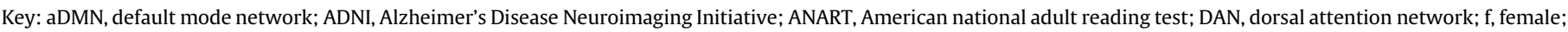

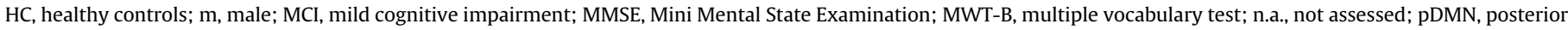
default mode network.

a Significantly different from ADNI-HC $(p<0.05)$.

b Significantly different from ISD-MCI.

(i.e., $A \beta$ deposition), we applied linear regression analyses, including the peak a(p)DMN-DAN anti-correlation as the dependent variable, and global AV-45 PET uptake as the predictor, controlling for age, gender, and education. Finally, we tested in linear regression analyses the association between the peak a(p)DMN-DAN anti-correlation and the mean (stationary) within-network functional connectivity, to assess whether the relationship between-network connectivity was influenced by the within-network integrity. To this end, we included the peak a(p)DMN-DAN anti-correlation as the dependent measure, and the CR proxy, mean within-network functional connectivity of the a(p)DMN and DAN as predictors, controlling for the memory composite score, age, gender the AV-45 PET status (ADNI sample) or the APOE genotype (ISD sample).

To eliminate methodological concerns, we tested whether head motion was in- or decreased during episodes of peak a(p)DMN-DAN anti-correlation versus the remaining resting-state scan. To this end, we applied Wilcoxon tests (due to nonnormal distribution of mean FD), testing differences between mean FD within time windows of peak a(p)DMN-DAN anti-correlation against mean FD within the remaining time windows. We further tested whether the peak a(p)DMN-DAN anti-correlation correlated with mean FD in that time windows.
Linear model assumptions were tested using the gvlma package in R. For all models reported, no significant deviations for linear model assumptions were found (alpha threshold $=0.05$ ). All statistical analyses were conducted in $\mathrm{R}$ statistical software (Version 2.13.2; $R$ Foundation for Statistical Computing, Vienna, Austria).

\section{Results}

\subsection{Sample characteristics}

Demographics, cognitive measures, and the a(p)DMN-DAN correlation coefficients for the ADNI and ISD samples are depicted in Table 1. In the ADNI sample, MCI patients were significantly younger than the HC subjects [ $\mathrm{t}(110)=2.80, p=0.006]$.

\subsection{Comparison of peak a(p)DMN-DAN anticorrelation between HC and MCI subjects}

In the ADNI sample, we tested whether MCI patients differed from $\mathrm{HC}$ regarding the a(p)DMN-DAN anti-correlation or within network FC measures. We found no differences in the a(p)DMNDAN anti-correlation between $\mathrm{HC}$ and $\mathrm{MCI}$. There were also

Table 2

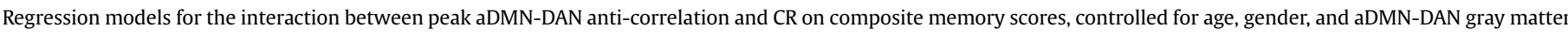
volume and mean FD

\begin{tabular}{|c|c|c|c|c|c|c|}
\hline Sample, model terms & $\begin{array}{l}\text { ADNI-HC } \\
\mathrm{B}(\mathrm{SE})\end{array}$ & $p$-value & $\begin{array}{l}\text { ADNI-MCI } \\
\text { B (SE) }\end{array}$ & $p$-value & $\begin{array}{l}\text { ISD-MCI } \\
\mathrm{B}(\mathrm{SE})\end{array}$ & $p$-value \\
\hline \multicolumn{7}{|l|}{ CR proxy: IQ } \\
\hline Intercept & $2.60(0.85)$ & 0.005 & $2.00(0.75)$ & 0.009 & $2.36(0.70)$ & 0.001 \\
\hline IQ X aDMN-DAN anticorrelation & $0.01(0.03)$ & 0.701 & $-0.03(0.02)$ & 0.030 & $0.01(0.01)$ & 0.015 \\
\hline IQ & $0.01(0.02)$ & 0.977 & $-0.02(0.01)$ & 0.017 & $0.27(0.12)$ & 0.031 \\
\hline aDMN-DAN anticorrelation & $-0.04(0.29)$ & 0.887 & $0.14(0.22)$ & 0.533 & $-0.70(0.33)$ & 0.036 \\
\hline \multicolumn{7}{|l|}{ CR proxy: years of education } \\
\hline Intercept & $2.85(1.38)$ & 0.049 & $0.25(0.83)$ & 0.776 & n.a & n.a \\
\hline Education $\mathrm{X}$ aDMN-DAN anticorrelation & $-0.03(0.10)$ & 0.733 & $0.14(0.04)$ & 0.002 & n.a & n.a \\
\hline Education & $-0.01(0.07)$ & 0.873 & $0.10(0.03)$ & 0.001 & n.a & n.a \\
\hline aDMN-DAN anticorrelation & $0.62(1.61)$ & 0.705 & $-2.65(0.77)$ & 0.001 & n.a & n.a \\
\hline
\end{tabular}

In the ADNI sample, we additionally controlled for AV45 uptake. In the ISD sample, we controlled for APOE carrier status ( $\varepsilon 4$ vs. other).

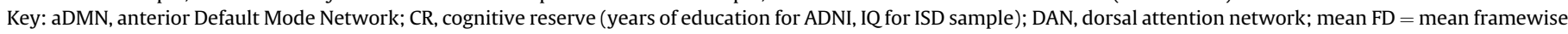
displacement (during the time windows of peak aDMN-DAN anti-correlation); n.a., not assessed. 
A

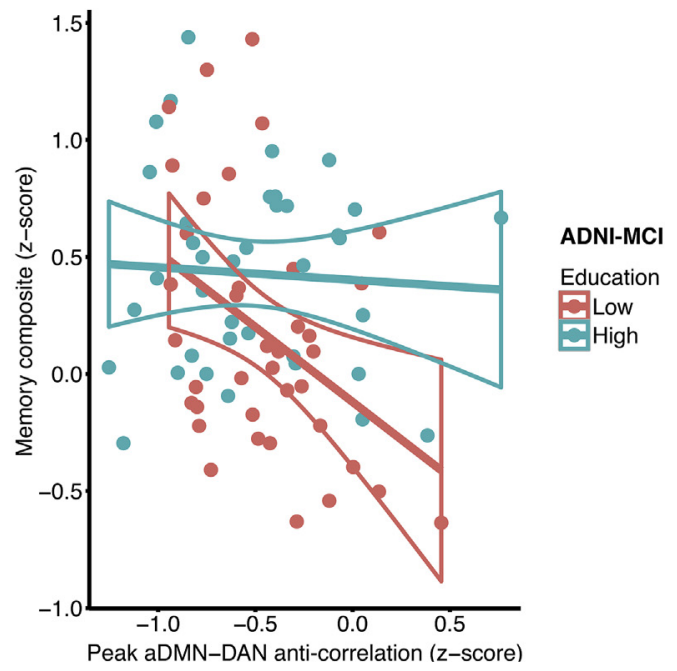

B

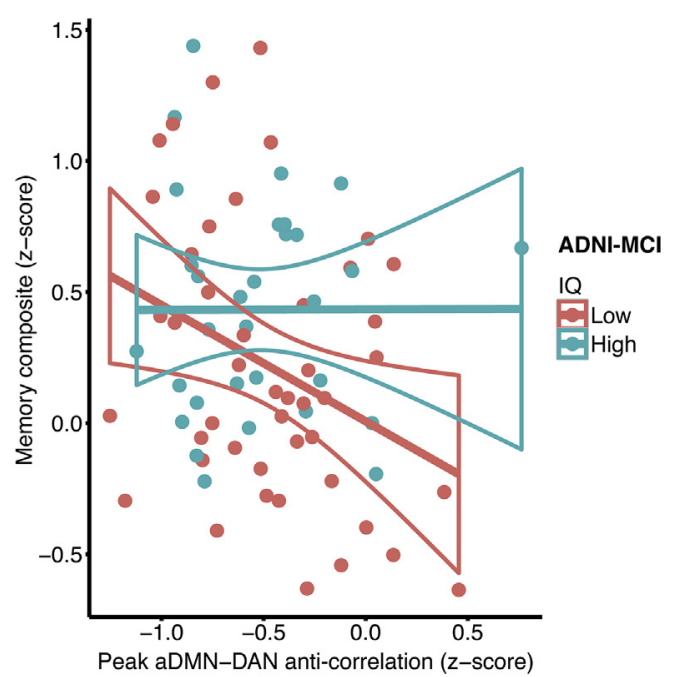

C

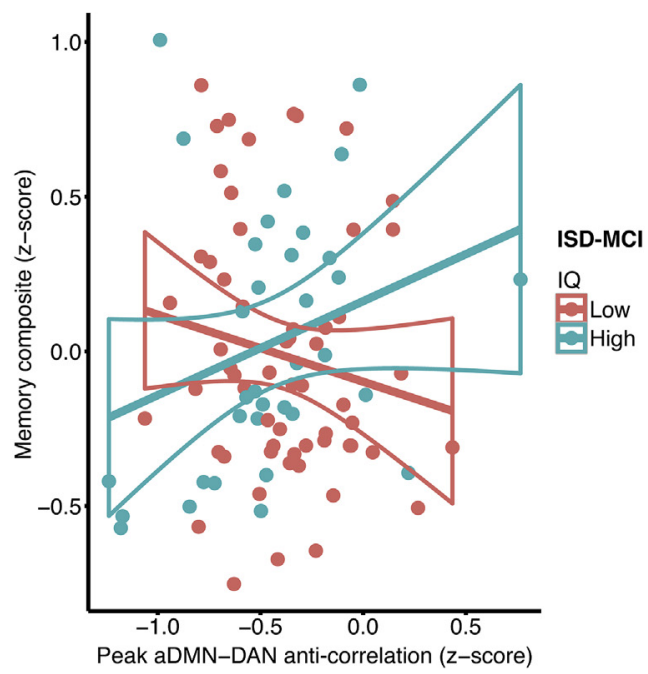

Fig. 3. Scatterplot for the interaction between peak aDMN-DAN anti-correlation and education (A) and IQ (B) for predicting the composite memory score in the ADNI-MCI sample. The correlation between the aDMN and the DAN is plotted against the composite memory score. Regression lines plus the $95 \%$ CIs are plotted for high and low CR (education or IQ) groups that were defined via median split for illustrational purposes. (C) Scatterplot for the interaction between IQ and peak aDMN-DAN anti-correlation for no observed differences, when $\mathrm{MCI} A \beta+$ and $\mathrm{MCI} A \beta$ - groups were separately compared to $\mathrm{HC}$, or to $\mathrm{HC} A \beta$ - only. Since $\mathrm{MCI}$ patients were significantly younger than $\mathrm{HC}$, we manually adjusted the a(p)DMN-DAN anti-correlations for age (see Table 1; details on the adjustment procedure can be found in the Supplementary data). Here, the a(p)DMN-DAN anti-correlation was numerically but not significantly decreased in $\mathrm{MCI}$ when compared to HC.

\section{3. $C R$ as moderator of the association between a(p)DMN-DAN anticorrelation and memory performance in $\mathrm{MCI}$}

For the ADNI study, the interaction effect of $\mathrm{CR} \times$ peak aDMN-DAN anti-correlation on the memory composite score was significant in $\mathrm{MCI}$ patients for different $\mathrm{CR}$ proxies [education: $\mathrm{t}(67)=3.176, \mathrm{~B}=0.14, \mathrm{SE}=0.04, p=0.002$; IQ: $\mathrm{t}(67)=-2.213$, $\mathrm{B}=-0.03, \mathrm{SE}=0.02, p=0.030]$. Fig. $3 \mathrm{~A}$ and $\mathrm{B}$ show that a decrease in the peak aDMN-DAN anti-correlation was associated with lower episodic memory composite scores in the low CR but not the high CR patients (groups are split at the median for illustrational purposes). Controlling for $A P O E$ genotype rather than $A \beta$ - status in the $\mathrm{MCI}$ patients did not change the result pattern. Congruent effects were found when entering static whole time course aDMN-DAN anticorrelation in the interaction term [education: $\mathrm{t}(67)=3.384$, $\mathrm{B}=0.18, \mathrm{SE}=0.05, p=0.001 ; \mathrm{IQ}: \mathrm{t}(67)=-2.083, \mathrm{~B}=-0.03$, $\mathrm{SE}=0.01, p=0.041]$. For the pDMN, the interaction effect of education $\times$ peak pDMN-DAN anti-correlation on the memory composite was not significant for both peak as well as static FC in $\mathrm{MCI}$. When tested in the HC group, we found no main effect of peak a(p)DMN-DAN anti-correlation on the memory composite nor was the interaction education or IQ $\times$ peak a(p)DMN-DAN anticorrelation significant (see Supplementary Fig. 1), suggesting reduced levels of peak aDMN-DAN anti-correlation to impact memory first at the level of $\mathrm{MCI}$. Cross-validation analysis in the ISD sample confirmed a significant interaction effect of IQ $\times$ peak aDMNDAN anti-correlation on the memory composite score in $\mathrm{MCI}$ $[\mathrm{t}(84)=2.477, \mathrm{~B}=0.01, \mathrm{SE}=0.01, p=0.015$; see Fig. $3 \mathrm{C}]$. Consistent with the results obtained in the ADNI study, a reduced peak aDMNDAN anti-correlation was associated with lower episodic memory composite scores at lower levels of IQ. A congruent interaction effect was found when using static whole time course aDMN-DAN anticorrelation $[\mathrm{t}(84)=2.498, \mathrm{~B} 0.02, \mathrm{SE}=0.008, p=0.014]$. Again, no significant interaction effect was observed between the IQ $\times$ peak pDMN-DAN anti-correlation on the composite memory score. For detailed regression statistics on interaction models please see Table 2.

Neither education (ADNI sample) nor IQ (ISD-sample) were correlated with the level of peak aDMN-DAN anti-correlation (ADNI: $r=-0.08, p=0.483$; ISD: $r=-0.03, p=0.79$ ), or pDMNDAN anti-correlation (ADNI: $\mathrm{r}=-0.19, p=0.15$; ISD: $\mathrm{r}=-0.08$, $p=0.45)$ suggesting that $C R$ does not alter the a(p)DMN-DAN anti-correlation itself.

\subsection{A $\beta$-status, education, and peak aDMN-DAN anti-correlation in $M C I(A D N I)$}

We next tested whether ( 1 ) greater $A \beta$ deposition was related to decreased levels of peak aDMN-DAN anti-correlation, or

predicting the composite memory score in the ISD-MCI sample. The correlation between the aDMN and the DAN is plotted against the composite memory score. For illustrational purposes, regression lines plus the $95 \%$ CIs are plotted for high IQ and low IQ groups that were again defined via median split. Abbreviations: aDMN, anterior default mode network; CR, cognitive reserve; DAN, dorsal attention network; ISD, Institute for Stroke and Dementia Research; $\mathrm{MCI}$, mild cognitive impairment. 


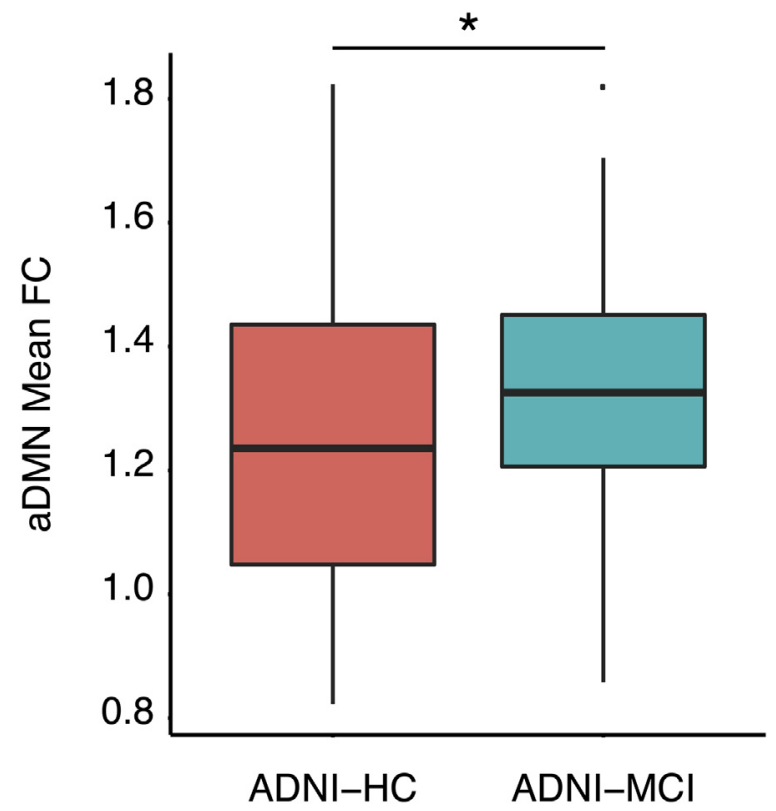

Fig. 4. Group comparison of the mean aDMN-FC between $\mathrm{HC}$ and $\mathrm{MCI}$ subjects of the ADNI sample. The group difference is significant at ${ }^{*} p<0.05$. Abbreviations: aDMN, anterior default mode network; ADNI, Alzheimer's Disease Neuroimaging Initiative; FC, functional connectivity; $\mathrm{HC}$, healthy controls; $\mathrm{MCI}$, mild cognitive impairment.

(2) whether the interaction $C R \times$ peak aDMN-DAN anti-correlation was different with regard to $A \beta$-status. In a linear regression model, global AV-45 PET levels did not predict peak aDMN-DAN anti-correlation [ $\mathrm{t}(73)=0.806, \mathrm{~B}=0.17, \mathrm{SE}=0.21, p=0.423$ ]. There were no differences in the effect of education $\times$ peak aDMN-DAN anti-correlation on memory between the $\mathrm{MCI}$ $\mathrm{A} \beta+$ and $\mathrm{MCI} A \beta$ - groups, suggesting that the moderating effect of $\mathrm{CR}$ on the association between peak aDMN-DAN anti-correlation and memory performance was not influenced by $A \beta$ status [3-way interaction: education: $\mathrm{t}(64)=-1.383, \mathrm{~B}=-0.26$, $\mathrm{SE}=0.11, p=0.17$; IQ: $\mathrm{t}(64)=-0.5, \mathrm{~B}=-0.013, \mathrm{SE}=0.027$, $p=0.618]$.

\subsection{Group differences in stationary within-network functional} connectivity and its association with peak a(p)DMN-DAN anticorrelation

When comparing the mean stationary functional connectivity within the networks (aDMN, pDMN, DAN) between MCI and HC of the ADNI sample, we found an increased aDMN-functional connectivity in ADNI-MCI compared to ADNI-HC $[\mathrm{t}(110)=-2.42$; $p=0.017$; see Fig. 4], but no differences in functional connectivity within the pDMN or DAN. Levels of within-network functional connectivity (DAN, aDMN, pDMN) were neither associated with the level of between-network anti-correlation nor with $\mathrm{CR}$ proxies in either sample.

\subsection{Peak aDMN-DAN anti-correlation and head motion}

To rule out that the possibility that our results were driven by excessive head motion, we first tested, whether head motion was different between time windows of peak aDMN-DAN anticorrelation and remaining time windows. In both samples, we found no significant differences between mean FD in the 10 time windows representing peak aDMN-DAN anti-correlation and mean FD in the remaining time windows (ADNI-MCI: $p=0.86$, ADNI-HC: $p=0.47$,
ISD-MCI: $p=0.12$ ). This suggests that head motion is not increased during episodes of peak aDMN-DAN anti-correlation. When testing whether the mean FD during peak aDMN-DAN anti-correlation was associated with the level of anti-correlation, we found no significant correlations (ADNI-MCI: $\mathrm{r}=0.01, p=0.92$, ADNI-HC: $\mathrm{r}=0.1, p=0.55$, ISD-MCI: $\mathrm{r}=0.15, p=0.17$ ). There were no significant differences in mean FD between HC and MCI patients in the ADNI sample ( $p=$ 0.63 ). $\mathrm{MCI}$ patients of the ISD sample showed significantly less head motion (i.e., smaller mean FD) than $\mathrm{MCl}$ patients of the ADNI sample $(\mathrm{U}=5464, p<0.001)$.

\section{Discussion}

The major finding of the present study was that in amnestic $\mathrm{MCI}$, higher levels of $C R$ proxies alleviated the association between lower aDMN-DAN anti-correlation and lower episodic memory performance. This effect was consistently detected in 2 independent samples of $\mathrm{MCI}$ patients, using different proxy measures for $\mathrm{CR}$ (education or IQ). Our findings suggest that higher levels of $\mathrm{CR}$ are associated with protective effects at the functional network level in $\mathrm{MCI}$ and allow maintenance of relatively high memory performance at low levels of aDMN-DAN crosstalk.

In line with our findings, protective effects of education and IQ in nondemented older adults have been suggested by several lines of research. First, older adults with higher levels of education and IQ show at any level of cognitive performance relatively more severe levels of gray matter atrophy (Bastin et al., 2012; Solé-Padullés et al., 2009), temporoparietal glucose hypometabolism (Bastin et al., 2012; Ewers et al., 2013; Morbelli et al., 2013), or white matter hyperintensities (Brickman et al., 2011; Teipel et al., 2009), suggesting that those with higher CR proxy levels can cope better with brain pathology. Second, higher levels of education and IQ are associated with a delayed onset of cognitive decline (Amieva et al., 2014; Hall et al., 2007; Vemuri et al., 2011) and conversion from $\mathrm{MCI}$ to AD dementia (Dekhtyar et al., 2015; Querbes et al., 2009), suggesting that individuals with higher CR show prolonged maintenance of preserved levels of cognitive performance. Consistent with these previous results, our current findings reveal protective effects of education and IQ at the functional network level in MCI, where education and IQ consistently moderate the effect of lower levels of aDMN-DAN anti-correlation on episodic memory.

We found in both MCI samples that the DMN contains subcomponents including and anterior and posterior part of the DMN (Damoiseaux et al., 2006, 2012; Di and Biswal, 2014; Jones et al., 2011, 2012). Interestingly, the anti-correlation between the aDMN-DMN rather than pDMN-DAN was associated with memory performance. Previous studies including voxel-based functional connectivity analyses reported the aDMN to be predominantly connected to the parietal regions of the DAN, but the pDMN to be primarily connected to the premotor cortex (Uddin et al., 2009). Thus, it is predominantly the aDMN which is associated with those brain regions of the DAN that are known to be involved in memory and attention (Kim, 2015; Kim et al., 2010; Vossel et al., 2014). Therefore, the interaction effect of CR $\times$ DMN-DAN anti-correlation on memory was selectively found for the aDMN component probably due to its stronger connectivity to memory-supporting brain structures.

The opposing activity pattern of the DAN and DMN has previously been proposed to subserve an adaptive mechanism to increase attention to external stimuli presented during a cognitive task and to reduce the interference from irrelevant processes (Andrews-Hanna et al., 2010; Fox et al., 2005; Fransson, 2005; Keller et al., 2015; Spreng et al., 2016). This DMN-DAN anti-correlation has been shown to be pathologically reduced in AD dementia but not yet at the stage of MCI (Wang et al., 2007; Zhu et al., 2016). Consistent with those results, we found a numerical but not 
significant reduction of the DMN-DAN anti-correlation in $\mathrm{MCI}$ when compared to HC. Together with our finding that at low levels of CR, weaker DMN-DAN anti-correlations are coupled to reduced memory performance only in $\mathrm{MCI}$, the current results suggest that the DMN-DAN anti-correlation becomes critical to sustain memory at a fragile state of the brain, such as observed in MCI at low levels of CR. During cognitive processes, such as successful memory performance, task-positive networks including the DAN are critically involved (Spreng et al., 2010; Whitfield-Gabrieli and Ford, 2012) and are thought to orchestrate efficient network activation in the brain during task performance (Fox et al., 2005). Especially in MCI, where medial temporal lobe structures become functionally isolated (Pasquini et al., 2015; Tahmasian et al., 2015), memory performance requires coordinated coupling of the DMN and DAN (Celone et al., 2006). Thus, in the presence of regionally circumscribed brain alterations in $\mathrm{MCI}$, the role of the anticorrelated DMN-DAN activity may become especially important to maintain brain processes of successful memory performance. Our finding of the interaction between CR proxies and aDMN-DAN anti-correlation suggests that higher levels of CR buffer the effects of relatively low anti-correlation of DAN and aDMN on memory at the stage of MCI.

Our results raise the question which functional brain changes underlie CR. In the current study, intranetwork connectivity of the aDMN was increased, consistent with previous findings of increased frontal connectivity in MCI and AD (Jones et al., 2011; McCarthy et al., 2014). Increased frontal connectivity and activation in $\mathrm{MCI}$ and $\mathrm{AD}$ has been previously considered as a mechanism of compensatory nature (Barulli and Stern, 2013; Jones et al., 2011; Zhou et al., 2010). However, in the present study, no association between aDMN and CR or the aDMN-DAN anti-correlation was found. Alternatively, the increased aDMN functional connectivity may reflect dedifferentiation of frontal lobe function in aging and neurodegenerative diseases (Dennis and Cabeza, 2011; Jones et al., 2011). Several previous studies have investigated the effect of $\mathrm{CR}$ on brain connectivity or task-related brain activation (Arenaza-Urquijo et al., 2013; Bosch et al., 2010; Marques et al., 2016; Stern et al., 2008) [for a review see (Arenaza-Urquijo et al., 2015; Stern, 2012)]. Higher CR proxies have been reported to be associated with higher temporal and parietal brain activation during a memory encoding task in AD (Solé-Padullés et al., 2009), and higher resting state frontal connectivity and parietal functional segregation in cognitively healthy subjects (Arenaza-Urquijo et al., 2013; Marques et al., 2016). Still, compensatory functional brain changes of $\mathrm{CR}$ that support memory performance in $\mathrm{MCI}$ are unknown. The current findings may provide a reference standard for the search of functional mechanisms that account for the moderating effects of $\mathrm{CR}$ in $\mathrm{MCI}$, i.e., the alleviated association between lower levels of the peak aDMN-DAN anti-correlation and memory impairment.

The present study included MCI subjects both with and without elevated risk for AD. Importantly, we found no effect of $A \beta$ status or ApoE4 carrier-status on the relationship between peak aDMN-DAN anti-correlation, CR proxies and memory performance. The DMNDAN anti-correlation is a fundamental feature of network interaction that is associated with cognition. The DMN-DAN has been found to be altered in many neurodegenerative and psychiatric disease and may thus not be specific to AD (Anticevic et al., 2012; Baggio et al., 2015). The current results in amnestic MCI suggest that lower levels of the aDMN-DAN anti-correlation are associated with memory impairment, which is alleviated by $C R$, regardless of the presence of $A D$ pathology.

Further, our samples included subjects with both single and multiple domain $\mathrm{MCI}$. It is an open question whether the $\mathrm{MCI}$ subtype influences the effects of CR proxies on the association between peak aDMN-DAN anti-correlation and memory. We did not assess this in the present study since a restriction of our sample to single or multiple domain $\mathrm{MCI}$ would have drastically reduced statistical power and thus limited the sensitivity to detect the hypothesized effects. We encourage future studies to test a possible effect of the MCI subtype in larger samples of single and multiple domain $\mathrm{MCI}$ patients.

For the present study, we used sliding time window analysis to capture states of peak aDMN-DAN anti-correlations. However, sliding time window functional connectivity analysis in restingstate fMRI is a relatively new method (Allen et al., 2014), and no standardized index to characterize dynamics of betweennetwork connectivity has been yet established [for review see (Hutchison et al., 2013b)]. In the present study, we used the peak anti-correlation of ICA derived time courses of the DMN components and DAN to measure states of high between-network anti-correlations. Alternative measures include the analysis of recurring patterns of functional connectivity derived from ROIto-ROI analyses or ICA which have been previously interpreted as mental states or modes of functional connectivity (Allen et al., 2014). However, the anti-correlation of the DMN components and DAN can be observed in multiple of such modes (Damaraju et al., 2014; Meskaldji et al., 2016). In the present study, the peak anticorrelation was therefore considered the index of choice. A previous study that analyzed such peak anti-correlations between DMN and DAN brain regions during resting-state has shown that this measure is a strong predictor of memory performance in $\mathrm{MCI}$ (Meskaldji et al., 2016). To assure that the index was not dependent on an arbitrary decision, such as the length of time intervals across which peak correlations were identified, we varied the interval length, observing virtually no change in the results. To assess whether our results were specific to our peak functional connectivity measure, we also computed the static whole time course aDMN-DAN anti-correlation, following the methodology of previous studies (Fox et al., 2005; Uddin et al., 2009). Here, we found consistent results, i.e., an interaction of aDMN-DAN anti-correlation $\times \mathrm{CR}$ proxies on memory performance across samples. These observations support the robustness of the current findings and that moderating effects of CR can be detected across methods.

It is important to keep in mind that the assessment of the anticorrelation between the DAN and DMN is sensitive to preprocessing steps including global signal regression (Murphy et al., 2009; Spreng et al., 2016; Weissenbacher et al., 2009) as well as physiological noise, which has been shown for both static and sliding time window functional connectivity analysis (Birn et al., 2014; Nikolaou et al., 2016). In the present study, we regressed out the signal of CSF and WM, without global signal removal from the BOLD signal time series. Regression-based removal of the CSF and WM signal fluctuation was previously shown as an effective control when compared against the standard of noise removal based on monitored breathing and cardiac activities (Chai et al., 2012; Chang and Glover, 2009). To further control for potential influence of movement, we could show that states of peak aDMNDAN anti-correlation were not associated with larger head motion. Moreover, we demonstrated that the anti-correlation between the aDMN and DAN was associated with memory performance in 2 independent MCI samples, rendering it unlikely that the levels of anti-correlation stemmed from nonneural sources. Supporting this notion, a previous resting-state EEG-fMRI study has shown changes in the BOLD DMN-DAN anti-correlation to be associated with simultaneous changes in EEG alpha band power (Chang et al., 2013). We caution, however, that the present study remains correlational in nature so that a clear cause and effect relationship cannot be discerned. 


\section{Conclusions}

In summary, the present study suggests that higher levels of education and IQ confer protective effects in $\mathrm{MCI}$, moderating the association between lower levels of aDMN-DAN anti-correlation and memory. The results provide a crucial support for the $C R$ hypothesis in that we show a cognitive benefit through altered association between functional network changes and cognitive outcome. Based on these results, questions on the search for compensatory brain mechanisms and therapeutic interventions to stimulate the moderating effects of CR at the functional level can be addressed in future studies.

\section{Disclosure statement}

The authors have no actual or potential conflicts of interest.

\section{Acknowledgements}

The research was funded by grants (to M. E.) of the LMUexcellent Initiative and the European Commission (PCIG12-GA-2012334259), Alzheimer's Forschung Initiative.

\section{Appendix A. Supplementary data}

Supplementary data associated with this article can be found, in the online version, at http://dx.doi.org/10.1016/j.neurobiolaging. 2016.11.013.

\section{References}

Allen, E.A., Damaraju, E., Plis, S.M., Erhardt, E.B., Eichele, T., Calhoun, V.D., 2014. Tracking whole-brain connectivity dynamics in the resting state. Cereb. Cortex 24, 663-676.

Amieva, H., Mokri, H., Le Goff, M., Meillon, C., Jacqmin-Gadda, H., Foubert-Samier, A., Orgogozo, J.M., Stern, Y., Dartigues, J.F., 2014. Compensatory mechanisms in higher-educated subjects with Alzheimer's disease: a study of 20 years of cognitive decline. Brain 137 (Pt 4), 1167-1175.

Andrews-Hanna, J.R., Reidler, J.S., Sepulcre, J., Poulin, R., Buckner, R.L., 2010. Functional-anatomic fractionation of the brain's default network. Neuron 65, 550-562.

Anticevic, A., Cole, M.W., Murray, J.D., Corlett, P.R., Wang, X.J., Krystal, J.H., 2012. The role of default network deactivation in cognition and disease. Trends Cognitive Sciences 16, 584-592.

Arenaza-Urquijo, E.M., Landeau, B., La Joie, R., Mevel, K., Mezenge, F., Perrotin, A., Desgranges, B., Bartres-Faz, D., Eustache, F., Chetelat, G., 2013. Relationships between years of education and gray matter volume, metabolism and functional connectivity in healthy elders. Neuroimage 83, 450-457.

Arenaza-Urquijo, E.M., Wirth, M., Chetelat, G., 2015. Cognitive reserve and lifestyle: moving towards preclinical Alzheimer's disease. Front. Aging Neurosci. 7, 134.

Ashburner, J., 2007. A fast diffeomorphic image registration algorithm. Neuroimage 38, 95-113.

Ashburner, J., Friston, K.J., 2005. Unified segmentation. Neuroimage 26, 839-851.

Baggio, H.C., Segura, B., Sala-Llonch, R., Marti, M.J., Valldeoriola, F., Compta, Y., Tolosa, E., Junque, C., 2015. Cognitive impairment and resting-state network connectivity in Parkinson's disease. Hum. Brain Mapp. 36, 199-212.

Barulli, D., Stern, Y., 2013. Efficiency, capacity, compensation, maintenance, plasticity: emerging concepts in cognitive reserve. Trends Cognitive Sciences 17, 502-509.

Bastin, C., Yakushev, I., Bahri, M.A., Fellgiebel, A., Eustache, F., Landeau, B., Scheurich, A., Feyers, D., Collette, F., Chetelat, G., Salmon, E., 2012. Cognitive reserve impacts on interindividual variability in resting-state cerebral metabolism in normal aging. Neuroimage 63, 713-722.

Birn, R.M., Cornejo, M.D., Molloy, E.K., Patriat, R., Meier, T.B., Kirk, G.R., Nair, V.A., Meyerand, M.E., Prabhakaran, V., 2014. The influence of physiological noise correction on test-retest reliability of resting-state functional connectivity. Brain Connect. 4, 511-522.

Bosch, B., Bartrés-Faz, D., Rami, L., Arenaza-Urquijo, E.M., Fernández-Espejo, D., Junqué, C., Solé-Padullés, C., Sánchez-Valle, R., Bargalló, N., Falcón, C., Molinuevo, J.L., 2010. Cognitive reserve modulates task-induced activations and deactivations in healthy elders, amnestic mild cognitive impairment and mild Alzheimer's disease. Cortex 46, 451-461.

Brickman, A.M., Siedlecki, K.L., Muraskin, J., Manly, J.J., Luchsinger, J.A., Yeung, L.K., Brown, T.R., DeCarli, C., Stern, Y., 2011. White matter hyperintensities and cognition: testing the reserve hypothesis. Neurobiol. Aging 32, 1588-1598.
Bright, P., Jaldow, E., Kopelman, M.D., 2002. The National Adult Reading Test as a measure of premorbid intelligence: a comparison with estimates derived from demographic variables. J. Int. Neuropsychol. Soc. 8, 847-854.

Calhoun, V.D., Adali, T., 2012. Multisubject independent component analysis of fMRI: a decade of intrinsic networks, default mode, and neurodiagnostic discovery. IEEE Rev. Biomed. Eng. 5, 60-73.

Carbonell, F., Bellec, P., Shmuel, A., 2014. Quantification of the impact of a confounding variable on functional connectivity confirms anti-correlated networks in the resting-state. Neuroimage 86, 343-353.

Celone, K.A., Calhoun, V.D., Dickerson, B.C. Atri, A., Chua, E.F., Miller, S.L, DePeau, K. Rentz, D.M., Selkoe, D.J., Blacker, D., Albert, M.S., Sperling, R.A., 2006. Alterations in memory networks in mild cognitive impairment and Alzheimer's disease: an independent component analysis. J. Neurosci. 26, 10222-10231.

Chai, X.J., Castanon, A.N., Ongur, D., Whitfield-Gabrieli, S., 2012. Anticorrelations in resting state networks without global signal regression. Neuroimage 59, $1420-1428$.

Chai, X.J., Ofen, N., Gabrieli, J.D., Whitfield-Gabrieli, S., 2014. Selective development of anticorrelated networks in the intrinsic functional organization of the human brain. J. Cogn. Neurosci. 26, 501-513.

Chang, C., Glover, G.H., 2009. Effects of model-based physiological noise correction on default mode network anti-correlations and correlations. Neuroimage 47, $1448-1459$

Chang, C., Glover, G.H., 2010. Time-frequency dynamics of resting-state brain connectivity measured with fMRI. Neuroimage 50, 81-98.

Chang, C., Liu, Z., Chen, M.C., Liu, X., Duyn, J.H., 2013. EEG correlates of time-varying BOLD functional connectivity. Neuroimage 72, 227-236.

Cole, M.W., Repovs, G., Anticevic, A., 2014. The frontoparietal control system: a central role in mental health. Neuroscientist 20, 652-664.

Crane, P.K., Carle, A., Gibbons, L.E., Insel, P., Mackin, R.S., Gross, A., Jones, R.N. Mukherjee, S., Curtis, S.M., Harvey, D., Weiner, M., Mungas, D. Alzheimer's Disease Neuroimaging Initiative, 2012. Development and assessment of a composite score for memory in the Alzheimer's Disease Neuroimaging Initiative (ADNI). Brain Imaging Behav. 6, 502-516.

Damaraju, E., Allen, E.A., Belger, A., Ford, J.M., McEwen, S., Mathalon, D.H., Mueller, B.A. Pearlson, G.D., Potkin, S.G., Preda, A., Turner, J.A., Vaidya, J.G., van Erp, T.G. Calhoun, V.D., 2014. Dynamic functional connectivity analysis reveals transient states of dysconnectivity in schizophrenia. Neuroimage Clin. 5, 298-308.

Damoiseaux, J.S., Prater, K.E., Miller, B.L., Greicius, M.D., 2012. Functional connectivity tracks clinical deterioration in Alzheimer's disease. Neurobiol. Aging 33 828.e19-828.e30.

Damoiseaux, J.S., Rombouts, S.A., Barkhof, F., Scheltens, P., Stam, C.J., Smith, S.M. Beckmann, C.F., 2006. Consistent resting-state networks across healthy subjects. Proc. Natl. Acad. Sci. U. S. A. 103, 13848-13853.

Dekhtyar, S., Wang, H.X., Scott, K., Goodman, A., Koupil, I., Herlitz, A., 2015. A lifecourse study of cognitive reserve in dementia-from childhood to old age. Am. J. Geriatr. Psychiatry 23, 885-896.

Dennis, N.A., Cabeza, R., 2011. Age-related dedifferentiation of learning systems: an fMRI study of implicit and explicit learning. Neurobiol. Aging 32, 231.e17-231.e30.

Di, X., Biswal, B.B., 2014. Modulatory interactions between the default mode network and task positive networks in resting-state. PeerJ 2, e367.

Erhardt, E.B., Rachakonda, S., Bedrick, E.J., Allen, E.A., Adali, T., Calhoun, V.D., 2011 Comparison of multi-subject ICA methods for analysis of fMRI data. Hum. Brain Mapp. 32, 2075-2095.

Esiri, M.M., Chance, S.A., 2012. Cognitive reserve, cortical plasticity and resistance to Alzheimer's disease. Alzheimers Res. Ther. 4, 7.

Ewers, M., Insel, P.S., Stern, Y., Weiner, M.W. Alzheimer's Disease Neuroimaging Initiative, 2013. Cognitive reserve associated with FDG-PET in preclinical Alzheimer disease. Neurology 80, 1194-1201.

Fox, M.D., Snyder, A.Z., Vincent, J.L., Corbetta, M., Van Essen, D.C., Raichle, M.E., 2005. The human brain is intrinsically organized into dynamic, anticorrelated functional networks. Proc. Natl. Acad. Sci. U. S. A. 102, 9673-9678.

Fransson, P., 2005. Spontaneous low-frequency BOLD signal fluctuations: an fMR investigation of the resting-state default mode of brain function hypothesis. Hum. Brain Mapp. 26, 15-29.

Franzmeier, N., Unterauer, E., Ewers, M., Düring, M., Müller, C., Ruiescu, D., ErtlWagner, B., Teipel, S., Fuchs, C., Coloma Andrews, L., Dichgans, M., Bürger, K. 2016. Effects of age, APOE $\varepsilon 4$, cognitive reserve and hippocampal volume on cognitive intervention outcome in amnestic mild cognitive impairment J. Alzheimer's Dis. Parkinsonism 6,1-7.

Hall, C.B., Derby, C., LeValley, A., Katz, M.J., Verghese, J., Lipton, R.B., 2007. Education delays accelerated decline on a memory test in persons who develop dementia. Neurology 69, 1657-1664.

Hampson, M., Driesen, N., Roth, J.K., Gore, J.C., Constable, R.T., 2010. Functional connectivity between task-positive and task-negative brain areas and its relation to working memory performance. Magn. Reson. Imaging 28, 1051-1057.

Hessler, J., J, T. Kurz, A., Bickel, H., 2013. The MWT-B as an estimator of premorbid intelligence in MCI and dementia. Z. für. Neuropsychol. 24, 129-137.

Hutchison, R.M., Womelsdorf, T., Allen, E.A., Bandettini, P.A., Calhoun, V.D. Corbetta, M., Della Penna, S., Duyn, J.H., Glover, G.H., Gonzalez-Castillo, J., Handwerker, D.A., Keilholz, S., Kiviniemi, V., Leopold, D.A., de Pasquale, F. Sporns, O., Walter, M., Chang, C., 2013a. Dynamic functional connectivity: promise, issues, and interpretations. Neuroimage 80, 360-378.

Hutchison, R.M., Womelsdorf, T., Gati, J.S., Everling, S., Menon, R.S., 2013b. Restingstate networks show dynamic functional connectivity in awake humans and anesthetized macaques. Hum. Brain Mapp. 34, 2154-2177. 
Jones, D.T., Machulda, M.M., Vemuri, P., McDade, E.M., Zeng, G., Senjem, M.L., Gunter, J.L., Przybelski, S.A., Avula, R.T., Knopman, D.S., Boeve, B.F., Petersen, R.C., Jack Jr., C.R., 2011. Age-related changes in the default mode network are more advanced in Alzheimer disease. Neurology 77, 1524-1531.

Jones, D.T., Vemuri, P., Murphy, M.C., Gunter, J.L., Senjem, M.L., Machulda, M.M., Przybelski, S.A., Gregg, B.E., Kantarci, K., Knopman, D.S., Boeve, B.F., Petersen, R.C., Jack Jr., C.R., 2012. Non-stationarity in the "resting brain's" modular architecture. PLoS One 7, e39731.

Keller, J.B., Hedden, T., Thompson, T.W., Anteraper, S.A., Gabrieli, J.D., WhitfieldGabrieli, S., 2015. Resting-state anticorrelations between medial and lateral prefrontal cortex: association with working memory, aging, and individual differences. Cortex 64, 271-280.

Kelly, A.M., Uddin, L.Q., Biswal, B.B., Castellanos, F.X., Milham, M.P., 2008. Competition between functional brain networks mediates behavioral variability. Neuroimage 39, 527-537.

Kim, H., 2015. Encoding and retrieval along the long axis of the hippocampus and their relationships with dorsal attention and default mode networks: the HERNET model. Hippocampus 25, 500-510.

Kim, H., Daselaar, S.M., Cabeza, R., 2010. Overlapping brain activity between episodic memory encoding and retrieval: roles of the task-positive and tasknegative networks. Neuroimage 49, 1045-1054.

Kragel, J.E., Polyn, S.M., 2015. Functional interactions between large-scale networks during memory search. Cereb. Cortex 25, 667-679.

Laird, A.R., Fox, P.M., Eickhoff, S.B., Turner, J.A., Ray, K.L., McKay, D.R., Glahn, D.C., Beckmann, C.F., Smith, S.M., Fox, P.T., 2011. Behavioral interpretations of intrinsic connectivity networks. J. Cogn. Neurosci. 23, 4022-4037.

Landau, S.M., Breault, C., Joshi, A.D., Pontecorvo, M., Mathis, C.A., Jagust, W.J., Mintun, M.A. Alzheimer's Disease Neuroimaging Initiative, 2013. Amyloid-beta imaging with Pittsburgh compound B and florbetapir: comparing radiotracers and quantification methods. J. Nucl. Med. 54, 70-77.

Landsiedel, J., Gilbert, S.J., 2015. Creating external reminders for delayed intentions: dissociable influence on "task-positive" and "task-negative" brain networks. Neuroimage 104, 231-240.

Luck, T., Riedel-Heller, S., Wiese, B., 2009. CERAD-NP-Testbatterie: Alters-, geschlechts- und bildungsspezifischen Normen ausgewählter Subtests. Z. Gerontol. Geriatr. 42, 372-384.

Mak, H.K., Zhang, Z., Yau, K.K., Zhang, L., Chan, Q., Chu, L.W., 2011. Efficacy of voxelbased morphometry with DARTEL and standard registration as imaging biomarkers in Alzheimer's disease patients and cognitively normal older adults at 3.0 Tesla MR imaging. J. Alzheimer's Dis. JAD 23, 655-664.

Marques, P., Moreira, P., Magalhaes, R., Costa, P., Santos, N., Zihl, J., Soares, J., Sousa, N., 2016. The functional connectome of cognitive reserve. Hum. Brain Mapp. 37, 3310-3322.

McCarthy, P., Benuskova, L., Franz, E.A., 2014. The age-related posterior-anterior shift as revealed by voxelwise analysis of functional brain networks. Front. Aging Neurosci. 6, 301.

Meng, X., D'Arcy, C., 2012. Education and dementia in the context of the cognitive reserve hypothesis: a systematic review with meta-analyses and qualitative analyses. PLoS One 7, e38268.

Meskaldji, D.-E., Preti, M.G., Bolton, T.A.W., Montandon, M.-L., Rodriguez, C., Morgenthaler, S., Giannakopoulos, P., Haller, S., Van De Ville, D., 2016. Prediction of long-term memory scores in $\mathrm{MCI}$ based on resting-state fMRI. Neuroimage Clin. 12, 785-795.

Morbelli, S., Perneczky, R., Drzezga, A., Frisoni, G.B., Caroli, A., van Berckel, B.N., Ossenkoppele, R., Guedj, E., Didic, M., Brugnolo, A., Naseri, M., Sambuceti, G., Pagani, M., Nobili, F., 2013. Metabolic networks underlying cognitive reserve in prodromal Alzheimer disease: a European Alzheimer disease consortium project. J. Nucl. Med. 54, 894-902.

Murphy, K., Birn, R.M., Handwerker, D.A., Jones, T.B., Bandettini, P.A., 2009. The impact of global signal regression on resting state correlations: are anticorrelated networks introduced? Neuroimage 44, 893-905.

Nikolaou, F., Orphanidou, C., Papakyriakou, P., Murphy, K., Wise, R.G., Mitsis, G.D., 2016. Spontaneous physiological variability modulates dynamic functional connectivity in resting-state functional magnetic resonance imaging. Philos. Trans. A. Math. Phys. Eng. Sci. 374.

Nucci, M., Mapelli, D., Mondini, S., 2012. Cognitive reserve index questionnaire (CRIq): a new instrument for measuring cognitive reserve. Aging Clin. Exp. Res. 24, 218-226.

Pasquini, L., Scherr, M., Tahmasian, M., Meng, C., Myers, N.E., Ortner, M., Muhlau, M., Kurz, A., Forstl, H., Zimmer, C., Grimmer, T., Wohlschlager, A.M., Riedl, V., Sorg, C., 2015. Link between hippocampus' raised local and eased global intrinsic connectivity in AD. Alzheimers Dement 11, 475-484.

Petersen, R.C., 2004. Mild cognitive impairment as a diagnostic entity. J. Intern. Med. 256, 183-194.

Petersen, R.C., Aisen, P.S., Beckett, L.A., Donohue, M.C., Gamst, A.C., Harvey, D.J., Jack Jr., C.R., Jagust, W.J., Shaw, L.M., Toga, A.W., Trojanowski, J.Q., Weiner, M.W., 2010. Alzheimer's disease neuroimaging initiative (ADNI): clinical characterization. Neurology 74, 201-209.

Power, J.D., Barnes, K.A., Snyder, A.Z., Schlaggar, B.L., Petersen, S.E., 2012. Spurious but systematic correlations in functional connectivity MRI networks arise from subject motion. Neuroimage 59, 2142-2154.

Querbes, O., Aubry, F., Pariente, J., Lotterie, J.A., Demonet, J.F., Duret, V., Puel, M., Berry, I., Fort, J.C., Celsis, P., 2009. Early diagnosis of Alzheimer's disease using cortical thickness: impact of cognitive reserve. Brain 132 (Pt 8), 2036-2047.
Rentz, D.M., Locascio, J.J., Becker, J.A., Moran, E.K., Eng, E., Buckner, R.L. Sperling, R.A., Johnson, K.A., 2010. Cognition, reserve, and amyloid deposition in normal aging. Ann. Neurol. 67, 353-364.

Rozzini, L., Vicini Chilovi, B., Bertoletti, E., Conti, M., Delrio, I., Trabucchi, M. Padovani, A., 2008. The importance of Alzheimer disease assessment scalecognitive part in predicting progress for amnestic mild cognitive impairment to Alzheimer disease. J. Geriatr. Psychiatry Neurol. 21, 261-267.

Sadaghiani, S., Poline, J.B., Kleinschmidt, A., D’Esposito, M., 2015. Ongoing dynamics in large-scale functional connectivity predict perception. Proc. Natl. Acad. Sci. U. S. A. $112,8463-8468$.

Smith, S.M., Fox, P.T., Miller, K.L., Glahn, D.C., Fox, P.M., Mackay, C.E., Filippini, N., Watkins, K.E., Toro, R., Laird, A.R., Beckmann, C.F., 2009. Correspondence of the brain's functional architecture during activation and rest. Proc. Natl. Acad. Sci. U. S. A. 106, 13040-13045.

Solé-Padullés, C., Bartrés-Faz, D., Junqué, C., Vendrell, P., Rami, L., Clemente, I.C. Bosch, B., Villar, A., Bargalló, N., Jurado, M.A., Barrios, M., Molinuevo, J.L., 2009 Brain structure and function related to cognitive reserve variables in normal aging, mild cognitive impairment and Alzheimer's disease. Neurobiol. Aging 30, $1114-1124$

Spreng, R.N., Stevens, W.D., Chamberlain, J.P., Gilmore, A.W., Schacter, D.L., 2010 Default network activity, coupled with the frontoparietal control network, supports goal-directed cognition. Neuroimage 53, 303-317.

Spreng, R.N., Stevens, W.D., Viviano, J.D., Schacter, D.L., 2016. Attenuated anticorrelation between the default and dorsal attention networks with aging: evidence from task and rest. Neurobiol. Aging 45, 149-160.

Stern, Y., 2006. Cognitive reserve and Alzheimer disease. Alzheimer Dis. Assoc Disord. 20 (3 Suppl 2), S69-S74.

Stern, Y., 2012. Cognitive reserve in ageing and Alzheimer's disease. Lancet Neurol. $11,1006-1012$.

Stern, Y., Zarahn, E., Habeck, C., Holtzer, R., Rakitin, B.C., Kumar, A., Flynn, J., Steffener, J., Brown, T., 2008. A common neural network for cognitive reserve in verbal and object working memory in young but not old. Cereb. Cortex 18 959-967.

Tahmasian, M., Pasquini, L., Scherr, M., Meng, C., Forster, S., Mulej Bratec, S., Shi, K. Yakushev, I., Schwaiger, M., Grimmer, T., Diehl-Schmid, J., Riedl, V., Sorg, C. Drzezga, A., 2015. The lower hippocampus global connectivity, the higher its local metabolism in Alzheimer disease. Neurology 84, 1956-1963.

Teipel, S., Grothe, M.J., Zhou, J., Sepulcre, J., Dyrba, M., Sorg, C., Babiloni, C., 2016 Measuring cortical connectivity in Alzheimer's disease as a brain neural network pathology: toward clinical applications. J. Int. Neuropsychol. Soc. 22 $138-163$.

Teipel, S.J., Meindl, T., Wagner, M., Kohl, T., Burger, K., Reiser, M.F., Herpertz, S. Moller, H.J., Hampel, H., 2009. White matter microstructure in relation to education in aging and Alzheimer's disease. J. Alzheimers Dis. 17, 571-583.

Tucker, A.M., Stern, Y., 2011. Cognitive reserve in aging. Curr. Alzheimer Res. 8 , $354-360$.

Uddin, L.Q., Kelly, A.M., Biswal, B.B., Castellanos, F.X., Milham, M.P., 2009. Functiona connectivity of default mode network components: correlation, anticorrelation, and causality. Hum. Brain Mapp. 30, 625-637.

Valenzuela, M.J., Sachdev, P., 2006. Brain reserve and dementia: a systematic review Psychol. Med. 36, 441-454.

Vemuri, P., Weigand, S.D., Przybelski, S.A., Knopman, D.S., Smith, G.E. Trojanowski, J.Q., Shaw, L.M., Decarli, C.S., Carmichael, O., Bernstein, M.A. Aisen, P.S., Weiner, M., Petersen, R.C., Jack Jr., C.R. Alzheimer's Disease Neuroimaging Initiative, 2011. Cognitive reserve and Alzheimer's disease biomarkers are independent determinants of cognition. Brain 134 (Pt 5) 1479-1492.

Vossel, S., Geng, J.J., Fink, G.R., 2014. Dorsal and ventral attention systems: distinct neural circuits but collaborative roles. Neuroscientist 20,150-159.

Wang, K., Liang, M., Wang, L., Tian, L., Zhang, X., Li, K., Jiang, T., 2007. Altered functional connectivity in early Alzheimer's disease: a resting-state fMRI study. Hum. Brain Mapp. 28, 967-978.

Weissenbacher, A., Kasess, C., Gerstl, F., Lanzenberger, R., Moser, E. Windischberger, C., 2009. Correlations and anticorrelations in resting-state functional connectivity MRI: a quantitative comparison of preprocessing strategies. Neuroimage 47, 1408-1416.

Whitfield-Gabrieli, S., Ford, J.M., 2012. Default mode network activity and connectivity in psychopathology. Annu. Rev. Clin. Psychol. 8, 49-76.

Wilson, R.S., Mendes De Leon, C.F. Barnes, L.L. Schneider, JA., Bienias, J.L., Evans, D.A., Bennett, D.A., 2002. Participation in cognitively stimulating activities and risk of incident Alzheimer disease. JAMA 287, 742-748.

Woods, S.P., Delis, D.C., Scott, J.C., Kramer, J.H., Holdnack, J.A., 2006. The California Verbal Learning Test-second edition: test-retest reliability, practice effects, and reliable change indices for the standard and alternate forms. Arch. Clin. Neuropsychol. 21, 413-420.

Zalesky, A., Fornito, A., Cocchi, L., Gollo, L.L., Breakspear, M., 2014. Time-resolved resting-state brain networks. Proc. Natl. Acad. Sci. U. S. A. 111, 10341-10346.

Zhou, J., Greicius, M.D., Gennatas, E.D., Growdon, M.E., Jang, J.Y., Rabinovici, G.D. Kramer, J.H., Weiner, M., Miller, B.L., Seeley, W.W., 2010. Divergent network connectivity changes in behavioural variant frontotemporal dementia and Alzheimer's disease. Brain 133 (Pt 5), 1352-1367.

Zhu, H., Zhou, P., Alcauter, S., Chen, Y., Cao, H., Tian, M., Ming, D., Qi, H., Wang, X. Zhao, X., He, F., Ni, H., Gao, W., 2016. Changes of intranetwork and internetwork functional connectivity in Alzheimer's disease and mild cognitive impairment. J. Neural. Eng. 13, 046008. 